Bayard, Chantal et Laurence Charton. « Partenariat rémunéré entre une entreprise et une influenceuse sur les réseaux sociaux : entrecroisement des voix des femmes autour de l'alimentation des nourrissons. "Nouvelle

Revue Synergies Canada, №15 (2022)

\title{
Partenariat rémunéré entre une entreprise et une influenceuse sur les réseaux sociaux : entrecroisement des voix des femmes autour de l'alimentation des nourrissons
}

\author{
Chantal Bayard \\ Laurence Charton \\ Centre Urbanisation Culture Société \\ Institut national de la recherche scientifique \\ Canada
}

Les réseaux socionumériques (RS) ont transformé le rapport des femmes enceintes et nouvellement mères à l'alimentation des nourrissons. Les récentes recherches sur l'allaitement (Marcon et al., 2018; Orchard et Nicholls, 2020 ; Locatelli, 2017) mentionnent notamment que les RS (Instagram, Facebook, Twitter) sont dorénavant des espaces qui permettent aux femmes : d'obtenir rapidement de l'information et des conseils pratiques, à toute heure du jour et de la nuit (Bridges et al., 2018); de partager leurs expériences (opinions, émotions, difficultés vécues, routine quotidienne) par la publication de textes/commentaires/images (Locatelli, 2017); et de trouver du soutien social et affectif de la part de personnes vivant des expériences similaires (NielaVilén et al., 2015 ; Regan et Brown, 2019 ; Skelton et al., 2018). Au fil des ans, les plateformes numériques ont aussi facilité la prise de parole et la présentation du point de vue des femmes qui militent en faveur de la « normalisation de l'allaitement ${ }^{1}$ " (lactivistes), une pratique qui suscite encore des réactions négatives sur les RS (Grant, 2016 ; Beach, 2017). Elles ont notamment contribué à rendre visible cette pratique dans les lieux publics (Bayard, 2014 ; Boyer, 2018), sur Internet et les RS (Buller, 2016 ; Boon et Pentney, 2015 ; Mecinska, 2018 ; Giles, 2018), que ce soit par la publication de brelfies - selfies d'allaitement - ou de commentaires en ligne. Les traces de ces prises de position sont visibles sous des hashtags comme \#normalizebreastfeeding sur Instagram (Locatelli, 2017) et \#brelfie sur Twitter (Beach, 2017). Enfin, on remarque aussi en ligne des actions organisées de sensibilisation du grand public à l'allaitement (allaite-in) ou de contestation de l'industrie (Morrissey et Kimball, 2017).

Si plusieurs recherches ont mis de l'avant les aspects positifs des RS pour les femmes notamment allaitantes, celles qui portent sur les femmes qui utilisent des préparations pour nourrissons se font plus rares. Pourtant, la présence de ces femmes est aussi observable sur les RS : groupes de discussion privés sur Facebook, partage d'images d'enfants nourris au biberon avec des préparations sur Instagram (\#bottlefeeding, \#formulafeeding \#formulafed), etc. Comme les femmes allaitantes, elles utilisent également Internet et les réseaux sociaux pour combler le peu d'informations obtenues sur les préparations pour nourrissons de la part des professionnels de la santé (médecins, sages-femmes, infirmières) (Appleton et al., 2018). Dans un article récent, Regan et Brown (2019) soulignent en ce sens que les femmes qui ont initié l'allaitement et qui participent à un groupe Facebook de soutien en ligne sont mal reçues et se sentent jugées lorsqu'elles partagent les raisons qui ont motivé leur transition vers les préparations pour nourrissons. Même si les RS sont les dépositaires des expériences sur l'alimentation des nourrissons, il semble demeurer un clivage entre les femmes qui allaitent exclusivement, conformément aux recommandations de l'Organisation mondiale de la santé (OMS, 2003; 2020) ${ }^{2}$, et les autres. Depuis les 20 dernières années, plusieurs chercheuses (Rippey, 2021 ; Jarty et Fournier, 2020 ; Baillargeon, 2004 ; Murphy, 2000 ; Wall, 2001 ; Kukla, 2006 ; Lee, 2008) ont d'ailleurs montré, à partir de l'analyse des discours religieux, politiques et de santé, comment la moralisation de l'acte de nourrir un nouveau-né s'est opérée à travers le temps, devenant ainsi l'un des jalons de " la mesure de la mère ». Callaghan et Lazard (2011) constatent en ce sens que les femmes qui participent à des forums sur Internet considèrent fréquemment l'allaitement comme étant « naturel », un « choix évident » à cause des propriétés du lait maternel et par extension être le " meilleur choix » en matière d'alimentation des nourrissons. Comparativement, et par opposition, l'alimentation au biberon est considérée comme une « deuxième option » non naturelle qui ne prend pas en considération le " meilleur intérêt de l'enfant » (p. 14-15). La polarisation des débats autour de l'alimentation des nourrissons, et l'opposition des différents modes d'alimentation, ont également été observées dans les échanges en ligne autour de l'allaitement dans l'espace public (Beach, 2017 ; Grant, 2016).

Des recherches récentes ont également porté sur les stratégies publicitaires en ligne des entreprises qui commercialisent des produits destinés aux femmes qui allaitent ou utilisent des préparations pour nourrissons. En effet, Internet et les RS sont devenus des dispositifs incontournables pour échanger et rejoindre directement les consommatrices (Leaver et al., 2020 ; Bernard, 2019). Sous les hashtags utilisés par les femmes pour partager leurs expériences (\#breastfeed, \#breastfeeding, \#normalizebreastfeeding, \#breastfeeding, \#breastmilk, \#breastfeedingmoms, \#breastfeedingsupport), on retrouve, par exemple, les publicités de produits associés à l'allaitement ou à l'alimentation au biberon: tire-lait, suppléments alimentaires, tétines, biberons, vêtements d'allaitement (Marcon et al., 2018 ; Moukarzel et al., 2020). Pour promouvoir leur marque et faire entendre leurs 
Bayard, Chantal et Laurence Charton. « Partenariat rémunéré entre une entreprise et une influenceuse sur les réseaux sociaux : entrecroisement des voix des femmes autour de l'alimentation des nourrissons. ॥ Nouvelle

Revue Synergies Canada, №15 (2022)

voix auprès de leur population cible, les compagnies qui commercialisent des préparations pour nourrissons multiplient ainsi les stratégies de marketing sur les RS: présence en ligne et création de contenu, développement d'applications mobiles, prise de contact direct avec la population cible, partenariats rémunérés avec des parents blogueurs, des influenceur.e.s et des personnalités publiques (Abrahams, 2012 ; Tanrikulu et al., 2020 ; van Tulleken et al., 2020). Dans cet article, nous nous intéresserons plus spécifiquement aux voix des internautes qui s'élèvent en réaction à un contenu de marque ${ }^{3}$ créé à des fins commerciales en partenariat avec une influenceuse, c'est-à-dire une personne qui :

[...] usually engage with positive self-branding strategies (as opposed to playing with notions of shame and scandal); manage a public visibility that is sustained and stable (as opposed to being briefly viral or transient); groom followers to consume their content aspirationally (as opposed to accumulating hatewatchers or audiences who tune in only with the desire to watch them fail or to gawk at them); and can parlay their high internet visibility into an income that is lucrative enough for full-time career (Abidin, citée dans Leaver et al., 2020, p. 106).

L'influenceuse agit en tant que porte-voix de l'entreprise auprès de ses d'abonné.e.s. Cette stratégie interpelle notamment les personnes et les institutions engagées dans la protection et la promotion de l'allaitement, car ce type de partenariat rémunéré contrevient au Code international de commercialisation des substituts du lait maternel (ci-après nommé « Le Code ») de l'OMS, adopté en 1981 et appuyé par 118 pays (OMS, 1981) dont le Canada (MSSS, 2018).

Les recherches précédentes sur l'alimentation des nourrissons et les RS suggèrent que les espaces numériques peuvent être appréhendés comme les dépositaires des voix des femmes qui s'expriment de manières plurielles (écrites, photographies, vidéos, dessins, graphiques, mèmes, etc.), selon des intensités variables (directes, puissantes, représentées) et avec des visées multiples (réconforter, contester, partager, rencontrer, débattre, vendre, etc.). Selon Rabatel (2012), la notion de voix peut être saisie à partir de l'analyse des relations entre un locuteur et un énonciateur. Ainsi, les voix sont " celles qu'on peut ramener à un locuteur effectif, réel » (12), c'est-à-dire à une personne à qui l'on peut demander « des explications et des comptes » (Rabatel, 2012, p. 12). Un phénomène qu'il est possible d'observer dans les interactions entre les internautes sur les RS où les individus s'interpellent mutuellement. Toujours selon Rabatel (2012), les différentes formes d'expression des voix « participent à la construction d'une position énonciative, un sujet modal, un centre de perspective, elle renvoie à la notion d'énonciateur, solidaire de la notion de point de vue » (p. 12). Autrement dit, une locutrice est une personne qui émet, par une pluralité de moyens, un point de vue situé sur un sujet donné qui témoigne de son rapport au monde. Points de vue qui sont notamment construits à partir d'un assemblage de discours passés et actuels, des caractéristiques sociales (âge, genre, classe, éducation, race) de la personne et de ses expériences. Au même titre que les « voix sonores », les voix dont il est question indexent « des attitudes, des postures, des émotions et des identités » (Arnold, 2016, p. 713). Au cœur des interactions sociales, les voix rendent ainsi visibles les rapports de pouvoir et les inégalités sociales (Charton et de Pierrepont, 2018). En ce sens, les énonciations des voix sur les RS sont révélatrices de l'imbrication de l'individuel et du collectif, du personnel et du politique (Hill Collins, 2016).

Dans cet article, l'analyse des voix portera plus spécifiquement sur les réactions des internautes sur les RS Facebook et Instagram à la suite de la publication d'un billet de blogue rédigé par une influenceuse québécoise (Marilou Bourdon, Trois fois par jour), en partenariat avec une entreprise qui commercialise des préparations pour nourrissons (Enfamil Canada). Dans un contexte québécois où l'allaitement est par ailleurs recommandé par les autorités de santé publique, comment ce type de partenariat rémunéré, entre une influenceuse "vedette " (cf. ci-dessous) et une entreprise commerciale, est-il accueilli par les abonnées et les consommatrices des pages Facebook et Instagram de l'entreprise Trois fois par jour? Quelles sont les voix qui émergent autour de l'alimentation des nourrissons à travers les commentaires des utilisatrices (il s'agit essentiellement de femmes)? Comment les voix de ces femmes s'entrecroisent et s'entrechoquent, se répondent/s'opposent/s'unissent-elles? Enfin, que nous disent ces arguments sur les normes entourant l'alimentation des nourrissons? Nous exposerons d'abord la méthodologie utilisée dans le cadre de cette recherche pour ensuite présenter les trois voix dominantes qui émergent de notre corpus, soit « les voix du mécontentement ou de la contestation », « les voix de l'expérience » et « les voix de l'approbation ». L'article se termine par une discussion autour des enjeux relatifs à la prise de parole des femmes sur l'alimentation des nourrissons sur les réseaux socionumériques. 
Bayard, Chantal et Laurence Charton. « Partenariat rémunéré entre une entreprise et une influenceuse sur les réseaux sociaux : entrecroisement des voix des femmes autour de l'alimentation des nourrissons. ॥ Nouvelle

Revue Synergies Canada, №15 (2022)

\section{Méthodologie}

Dans cette partie, nous présentons le cas étudié, l'approche privilégiée, le corpus utilisé, de même que le profil des utilisatrices.

\section{Cas étudié}

Cet article porte sur les réactions exprimées par les internautes sur les plateformes Facebook et Instagram à la suite de la publication d'un billet de blogue par l'influenceuse québécoise Marilou Bourdon ${ }^{5}$ sur son site Trois fois par jour (ci-après nommé TFPJ ou Trois fois par jour $)^{6}$. Cette publication intitulée « Nourrir son bébé sans culpabilité » (25 juin 2019) a été réalisée dans le cadre d'un partenariat rémunéré7 avec l'entreprise Enfamil Canada $^{8}$. Le billet publié ${ }^{9}$ comprend 1089 mots, dont deux mentions explicites à Enfamil Canada apparaissant en fin de publication avec un hyperlien menant vers le site internet de la compagnie. Le billet publié est accompagné d'une photographie de Marilou Bourdon tenant sa fille dans les bras. Ce billet, écrit au « Je », mentionne notamment les difficultés d'allaitement rencontrées par la blogueuse, sa philosophie en matière d'alimentation, l'importance de la liberté de choisir des mères ainsi que les raisons qui l'ont mené à utiliser des préparations pour nourrissons. Dans les publications Facebook et Instagram, on retrouve un extrait choisi du billet de blogue (224 mots), la photographie de l'influenceuse, de même que la mention de l'entreprise (tag) Enfamil Canada.

\section{Approche}

Dans le cadre de cette recherche, nous avons privilégié une approche qualitative pour dégager les discours qui émergent sur l'alimentation des nourrissons et le partenariat rémunéré à partir des énoncés écrits (commentaires), des émoticônes ${ }^{10}$ et des hashtags ${ }^{11}$ mobilisés par les utilisatrices. Les données ont été collectées, dénominalisées et numérotées, par la première autrice de l'article entre le 4 et le 7 mai 2020 . Elles ont été codées et analysées à partir d'une approche inductive à l'aide du logiciel N'Vivo 12. L'objectif principal consistait à dégager les discours qui émergent des commentaires générés à la suite de la publication du billet de blogue sur les plateformes Facebook et Instagram de TFPJ. L'analyse de discours s'appuie sur la prémisse que « les mots que nous choisissons pour parler de quelque chose, et la manière dont ils sont prononcés ou écrits, façonnent notre rapport au monde et notre manière de l'expérimenter » (Willig, 2014, notre traduction). Les RS, comme médiateurs de discours, influencent les façons de dire et d'exprimer sa voix. Ainsi, leur programmation, leur fonctionnement, les conditions d'utilisation, le nombre de mots permis pour s'exprimer, le type d'images autorisées sont quelques exemples d'éléments qui structurent le mode d'expression et les interactions selon les plateformes (Gibbs et al., 2015).

Notre approche comporte cependant quelques limites. Nous n'excluons pas que certains des commentaires aient été formulés par les entreprises TFPJ et Enfamil Canada ou par des personnes qui leur sont associées. II est également possible que des commentaires aient été formulés par des personnes de l'entourage (ami.es, famille) de l'influenceuse, car nous avons remarqué que son conjoint est intervenu sous la publication Instagram. De plus, nos analyses nous indiquent qu'une vingtaine d'utilisatrices interviennent sous au moins deux des trois publications du partenariat rémunéré (voir la note 7) et que, sous la publication Facebook à l'étude (billet de blogue), une quarantaine de personnes expriment leurs points de vue à plusieurs reprises (entre 2 et 7 commentaires) en faveur (18) ou en défaveur (23) de ce partenariat. Ces deux derniers éléments laissent entrevoir la possibilité d'une action de contestation organisée, une stratégie couramment utilisée sur les RS.

\section{Corpus}

La publication « Nourrir son bébé sans culpabilité » a suscité de nombreuses interactions sur les RS à la date du 8 avril 2020 : 1) 3,2 k réactions, 273 partages et 641 commentaires sur Facebook ; 2) 5063 réactions et 207 commentaires sur Instagram ${ }^{12}$.

Les deux publications cumulent un total de 848 commentaires. Cependant, notre corpus comprend 740 commentaires, soit 536 commentaires collectés manuellement sur Facebook et 204 sur Instagram. II subsiste donc un écart (108) entre le nombre de commentaires indiqué sur les publications (848) et le nombre de commentaires collecté (740). Pour expliquer cet écart, nous avançons l'hypothèse que plusieurs commentaires ont été effacés par le gestionnaire de la communauté de l'entreprise ou encore par les utilisatrices de la plateforme. Parmi les 740 commentaires, 6\% ont été rédigés par Trois fois par jour (32 sur Facebook et 12 sur Instagram). 
Bayard, Chantal et Laurence Charton. « Partenariat rémunéré entre une entreprise et une influenceuse sur les réseaux sociaux : entrecroisement des voix des femmes autour de l'alimentation des nourrissons. ॥ Nouvelle

Revue Synergies Canada, №15 (2022)

Au total, notre échantillon comprend 502 utilisatrices uniques, soit 334 personnes sur Facebook et 168 sur Instagram. II est possible d'identifier 3 personnes qui sont intervenues sur les deux plateformes. Cependant, ce nombre est à prendre avec précaution, car $36 \%$ des personnes sur Instagram utilisent des pseudonymes.

\section{Profil des utilisatrices}

Chaque profil des utilisateurs et des utilisatrices a été consulté pour établir un portrait à partir des données sociodémographiques accessibles. L'échantillon est composé à $99 \%$ de femmes $(499 / 502)^{13}$. À partir des profils consultés, nous estimons que l'âge moyen des internautes se situe autour de 30 ans. Près de trois femmes sur cinq internautes de Facebook et deux sur cinq d'Instagram ont indiqué avoir au moins un enfant. Les informations sociodémographiques étant parcellaires, les résultats présentés ne peuvent en aucun cas représenter le profil de l'ensemble des abonnées des comptes de TFPJ.

\section{Résultats}

Le partenariat rémunéré entre Enfamil Canada et Trois fois par jour a suscité des réactions polarisées de la part des utilisatrices des plateformes Facebook et Instagram de TFPJ. Sur Facebook, près de la moitié (48\%) des commentaires sous la publication portent sur le partenariat rémunéré (en faveur ou en défaveur) et leur insatisfaction quant à la gestion de la page par la marque TFPJ ; 19\% des commentaires concerne l'expérience des femmes en lien avec l'alimentation des nourrissons, alors que $2 \%$ des commentaires mixtes les deux arguments. Sur Instagram, les commentaires qui traitent du partenariat rémunéré sont moins nombreux (7\%) que ceux qui renvoient à l'expérience des femmes en lien avec l'alimentation de leurs nourrissons (24\%). Enfin, près du tiers des utilisatrices sur Facebook et plus des deux tiers sur Instagram formulent un commentaire succinct généralement pour signifier leur approbation avec le contenu du billet de blogue. À la lumière de ceci, notre analyse nous a permis de distinguer trois types de voix dominantes dans ce corpus, soit les voix du « mécontentement » ou de la « contestation », les voix de « l'expérience » et les voix de l' « approbation ».

\section{Les voix du mécontentement ou de la contestation}

La publication du billet de blogue de TFPJ sur les RS, plus spécifiquement son caractère commandité, est à la source de nombreux commentaires critiques de la part des utilisatrices. En effet, parmi les commentaires portant sur le partenariat rémunéré, $67 \%$ sont négatifs, $26 \%$ sont positifs et $6 \%$ sont neutres ou non classifiables. Les voix du « mécontentement ou de la contestation » à l'égard du partenariat rémunéré cherchent à mettre en lumière : 1) son caractère "illégal ou presque " en vertu du Code de l'OMS ; 2) réitérer l'importance de l'allaitement ; 3) révéler une « publicité déguisée »; et 4) dénoncer le « manque de transparence » de la marque TFPJ. Les commentaires positifs, moins nombreux, répondent à ces contestations en y opposant des arguments tels que le caractère volontaire de l'application du Code et la liberté de l'influenceuse de s'associer avec qui elle l'entend. Nous présentons les différents arguments dans les lignes qui suivent en accordant une prédominance aux voix du « mécontentement ou de la contestation ».

\section{Un partenariat illégal ou presque}

De nombreuses utilisatrices affirment que ce type de partenariat rémunéré est « presque illégal » ou « illégal ». La stratégie qui consiste à recourir à des influenceurs.ses pour promouvoir un produit est, selon elles, une façon pour l'industrie de contourner le Code qui stipule qu'il : « [...] ne devrait y avoir ni publicité, ni aucune forme de promotion auprès du grand public de produits visés par le présent Code » (OMS, 1981). Pour contrer ce qu'elles considèrent comme de la désinformation de la part de l'entreprise et de l'influenceuse, ces femmes utilisent différentes stratégies pour sensibiliser les internautes. Ainsi, elles : 1) partagent des liens internet menant à des sources jugées crédibles (exemple : Gouvernement du Québec) ; 2) signalent la publicité comme « illégale » à Facebook ; et 3) citent des extraits du Code.

Parce que c'est interdit de faire de la pub pour les substituts de lait maternel et donc que cette commandite a pas mal l'air d'un moyen détourné de le faire. En plus les commentaires qui contiennent le lien que j'ai mis se font supprimer depuis hier https://www.msss.gouv.qc.ca/professionnels/perinatalite/amis-des-bebes/code-international-decommercialisation-des-substituts-du-lait-maternel/ (FB, commentaire 37.1) 
Bayard, Chantal et Laurence Charton. « Partenariat rémunéré entre une entreprise et une influenceuse sur les réseaux sociaux : entrecroisement des voix des femmes autour de l'alimentation des nourrissons. ॥ Nouvelle

Revue Synergies Canada, №15 (2022)

D'autres voix, moins nombreuses, s'élèvent pour souligner que le Code n'est pas une loi, mais plutôt un code volontaire. Ce commentaire résume l'argument avancé par ces femmes : « Ce n'est pas illégal. Ce code n'est lié à aucune législation officielle. Ce sont des propositions, pas des articles de loi. De plus, c'est un code dont l'application doit être volontaire. On choisit de l'appliquer [...]. » $(F B, 37.2)$ Ces dernières suggèrent à leur tour que les précédentes désinforment les utilisatrices.

On voit donc que sous une même publication Facebook se négocie la recherche de ce qui est vrai, de la « bonne information ", à travers l'entrecroisement des voix des femmes qui s'opposent et se contredisent. Indépendamment de leur posture, ces femmes cherchent à mettre de l'avant leur vision respective du « meilleur » pour nourrir un nourrisson, les voix des premières (illégalité de promouvoir des préparations pour nourrissons) étant prédominantes par rapport aux secondes (Code volontaire) dans notre corpus.

\section{Les préparations pour nourrisson et le lait maternel : des aliments non équivalents}

Principalement sur Facebook, on remarque aussi que certaines femmes interviennent dans les commentaires pour contrecarrer un message sous-jacent du billet de blogue, à savoir que le lait maternel et les préparations pour nourrissons - appelés aussi « formules lactées », " lait en poudre », " lait artificiel » ou " formules » par les internautes - seraient présentés par l'influenceuse comme des aliments équivalents pour la santé de l'enfant.

[...] Parce que oui, on a le choix, mais non, ce n'est pas LE meilleur choix pour la santé du bébé. Faut quand même pas se mettre des œillères. Faire la promotion des formules lactées seraient de dire que c'est un choix équivalent à l'allaitement maternel (au sein ou au biberon) alors qu'il n'est est pas du tout le cas. [...] À eux [parents] de faire le choix qu'ils veulent, en toute connaissance de cause (FB, 1.7).

C'est certain que si l'intention est de nourrir ton enfant, c'est mission accomplie ... Pour ce qui est de donner le meilleur à ton enfant, malheureusement c'est l'échec! 웅 ; (FB, commentaire 62.5)

Quelques internautes appuient leur argumentation en invoquant, par exemple, que la prise de position de l'influenceuse contrevient aux messages portés actuellement par les autorités de santé publique (Santé Canada, OMS, 2020) qui recommandent l'allaitement exclusif jusqu'à six mois.

Ce type d'intervention, qui semble à première vue s'inscrire dans une volonté de rectifier une information erronée, vient en effet renforcer l'injonction d'allaiter et son caractère moral. Le lait maternel est ainsi présenté comme le « meilleur choix » pour alimenter son enfant et les parents qui décident autrement, indépendamment de leur situation, doivent être conscients de cela. Un rappel qui apparaît nécessaire pour ces femmes alors que dans les faits, nous le verrons dans la section suivante, il s'agit d'une information connue par les femmes enceintes (Vallières, 2021).

\section{Partenariat rémunéré : publicité déguisée?}

Dans leurs commentaires sur Facebook, un très grand nombre d'utilisatrices dénoncent également le caractère publicitaire du témoignage partagé par l'influenceuse dans son billet de blogue. Même si plusieurs d'entre elles qualifient le texte de " beau », " magnifique », " sincère » et " touchant », elles déplorent du même souffle ce type de marketing.

J'ai commencé à lire le texte en me disant: wow! C'est si bien dit de laisser les mamans faire leurs propres choix. Et ensuite POW! Une plug sur Enfamil ${ }^{-\odot}$. Ça enlève toute crédibilité ${ }^{*}$. (FB, commentaire 55.1)

Pour ces femmes, ce type de partenariat rémunéré fait « perdre tout son sens » ou « toute sa crédibilité » au message de l'influenceuse. Des utilisatrices soulignent qu'un témoignage " réellement » désintéressé sur la déculpabilisation des mères ne devrait pas être rémunéré par une compagnie qui vend des préparations pour nourrissons. II s'agit donc pour elles d'une «belle pub déguisée ». Certaines n'hésitent pas à remettre en question la " bonne foi » et l'authenticité de l'influenceuse et se disent " amèrement déçues " ou " fâchées » de cette association entre les marques. Elles appellent les autres utilisatrices à ne pas se laisser berner par cette publicité rédigée par une équipe de communication. 
Bayard, Chantal et Laurence Charton. « Partenariat rémunéré entre une entreprise et une influenceuse sur les réseaux sociaux : entrecroisement des voix des femmes autour de l'alimentation des nourrissons. » Nouvelle

Revue Synergies Canada, №15 (2022)

D'accord avec toi, malaise. Il y aurait eu moyen de partager cette belle expérience sans promouvoir une marque. Faire du placement de produit avec un sujet aussi sensible... je trouve ça ordinaire. (Instagram, commentaire 1)

Déculpabiliser les mères qui ne veulent ou ne peuvent pas allaiter est important. Ce n'est pas honteux de donner de la formule à son bébé. Par contre, ce texte est clairement une publicité déguisée de manière peu subtile. Pourquoi mettre le nom d'une marque de formule en particulier si le but est réellement empathique et désintéressé? (FB, commentaire 40)

Contrairement aux précédentes, certaines femmes soulignent que ce type de partenariat rémunéré n'a rien d'antinomique avec les pratiques habituelles de TFPJ. Elles soulignent dans leurs commentaires que l'entreprise s'associe régulièrement avec différentes marques (exemples : une entreprise de rénovation, une association qui fait la promotion des fromages du Québec, etc.). Pour ces femmes, le partenariat rémunéré entre ces deux marques permet à TFPJ d'offrir en retour des contenus gratuits sur ses plateformes. Elles ne voient pas non plus d'un mauvais œil le fait que Marilou Bourdon « gagne » sa vie de cette façon. Selon elles, l'influenceuse est libre de s'associer avec qui bon lui semble. Une utilisatrice de Facebook exprime :

Je ne comprends pas pourquoi certaines sont choquées parce qu'elle endosse une marque. Voyons, elle aurait pu tout simplement faire un bref texte pour vendre les mérites d'Enfamil [...] Vous voulez qu'elle continue à publier de nombreuses recettes, soit actives, près de sa clientèle? Je vous rappelle qu'à la base elle fait tout ça gratuitement!!!! [...] (FB, commentaire 14.12)

Certaines d'entre elles déplorent les critiques et jugements «disproportionnés » qui s'accumulent sous la publication Facebook. Elles vont jusqu'à dire que les réactions négatives provenant des autres utilisatrices sont des exemples du jugement entre les femmes, ce que dénonce justement le billet de blogue.

Enfin, d'autres utilisatrices s'interrogent sur le manque de clarté dans la présentation visuelle du partenariat rémunéré sur les RS, ce qui donne l'impression d'être une publicité déguisée.

Au-delà de tout ça, mon gros malaise, c'est que la collaboration n'est pas à aucun moment clairement identifié dans le texte sur le site, ni au début ni à la fin, plusieurs lecteurs l'ont lu sans s'en rendre compte. Et ça, ça manque clairement de transparence. [...] (FB, commentaire 1.9)

Cette impression d'avoir été « flouée » vécue par certaines utilisatrices s'explique probablement par la présentation de la publication sur les RS. Sur Facebook, par exemple, la mention " partenariat rémunéré » n'apparaît pas dans l'en-tête de la publication. Dans le texte d'accroche, la mention de la compagnie Enfamil Canada apparaît seulement lorsque l'utilisatrice clique sur « Afficher la suite ». Il est donc possible que certaines utilisatrices aient cliqué directement sur le lien qui menait au billet de blogue sans réaliser qu'il s'agissait d'un partenariat rémunéré.

Les voix des femmes s'entremêlent ici pour s'exprimer sur le contexte du partenariat en ligne. Certaines considèrent que le témoignage personnel de l'influenceuse sur le choix des femmes en matière d'alimentation des nourrissons souffre de son caractère publicitaire. La monétisation du message semble teinter la perception de ces femmes qui ont l'impression d'avoir été manipulées. Le manque de transparence dans l'affichage semble ajouté à ce sentiment. II en résulte une remise en doute de la sincérité de l'influenceuse, qualité mise de l'avant par la marque TFPJ. D'autres considèrent plutôt que ce partenariat n'entache pas son authenticité. Elles mettent plutôt l'accent sur la logique de bons procédés qui sous-tend ce type de partenariat, à savoir qu'elles consomment les publicités de TFPJ en échange de recettes gratuites. En ce sens, tout le monde y gagne.

\section{Manque de transparence de la marque TFPJ}

Plusieurs utilisatrices dénoncent également la gestion des RS de la marque TFPJ. En s'adressant directement à Marilou Bourdon ou au gestionnaire de sa communauté en ligne, elles manifestent leur déception ou leur colère face à la censure de leurs voix sous les publications Facebook et Instagram.

J'ai laissé un commentaire semblable qui a été effacé. J'espère sincèrement que ce n'est pas Marilou elle-même qui ait choisi de faire taire ceux qui soulignent [...] [que c'est] une publicité payée par et pour Enfamil [...] (Instagram, commentaire 2) 
Bayard, Chantal et Laurence Charton. « Partenariat rémunéré entre une entreprise et une influenceuse sur les réseaux sociaux : entrecroisement des voix des femmes autour de l'alimentation des nourrissons. ॥ Nouvelle

Revue Synergies Canada, №15 (2022)

Mon commentaire aussi a été censuré [...] Un gros bond vers le bas dans l'estime et plus jamais je n'achèterai quoi que ce soit de 3 fois par jour. (FB, commentaire 44.1)

La censure des voix des femmes qui contestent le partenariat conforte ces femmes dans leurs actions. Convaincues, elles persistent à faire entendre leurs voix en rédigeant un nouveau commentaire et en invitant (tag) une autre personne à en faire de même. Le gestionnaire de la communauté reste silencieux quant aux raisons qui motivent l'effacement des messages (exemples : informations inexactes, messages inappropriés, désaccords avec les internautes, etc.). Dans les faits, la marque Trois fois par jour intervient seulement à 2 reprises sous les commentaires laissés par les internautes qui sont mécontents ou contestent le partenariat. La première pour préciser une information et la seconde pour expliquer, par souci de transparence, les motivations de l'influenceuse :

Mes propos ne sont pas moins vraies parce que je reçois des sous pour payer mon épicerie en retour. Je n'aurais possiblement jamais pris le temps d'écrire ces lignes sur ce sujet si important, si ce n'était pas du fait qu'une marque (que je consommes déjà) m'a offert de prendre de mon temps de travail pour le faire. Parce qu'avec 2 enfants à la maison, à temps plein, j'ai moins de temps pour juste me laisser aller à l'écriture pour le plaisir. Et ça me manque. Bref, je gagne, ceux qui aiment ce texte gagnent, et les autres, et bien, ils ne perdent rien. J'étais la première à diaboliser la publicité alors sans rancune (mon magazine est $0 \%$ pub) mais je crois qu'il faut rester ouvert. (émo : cœur rouge) Si tu trouves mon texte poche, c'est autre chose (émos : trois bonhommes rire aux éclats - avec deux larmes)! Et tu aurais bien le droit.

Ce commentaire, qui semble avoir été rédigé par l'influenceuse, a suscité la colère de quelques internautes qui trouvent cette justification malhonnête. Pour ces femmes, Marilou Bourdon est une femme d'affaires prospère qui n'a pas besoin de s'associer à ce type d'entreprise pour promouvoir sa marque. Une femme dit : " "Payer ton épicerie et nourrir ta famille" arrête je vais pleurer. Tu devrais te lancer en politique Marilou! Franchement tu nous prends pour qui? " (FB, 30.8) Au contraire de réconcilier les utilisatrices, ce commentaire semble plutôt avoir eu pour effet de raffermir les positions.

Cette gestion de la communauté révèle l'existence des tensions qui existent entre un groupe d'utilisatrices et la promotion de ce type de produit (préparations pour nourrissons) par la marque. Certes, l'influenceuse a le pouvoir de diffuser et d'amplifier un message à des centaines de milliers d'abonnées et de restreindre la parole, mais à l'inverse, les utilisatrices peuvent contribuer à nuire à l'image de marque de l'entreprise en inondant ses RS de commentaires dénonçant ce partenariat. Ainsi, comme consommatrices, des utilisatrices en informent d'autres de leurs actions de se désabonner ou de cesser d'acheter les produits de TFPJ, et les invitent à en faire de même. Des commentaires auxquels TFPJ ne répond pas. D'autres femmes qui prennent la défense de l'influenceuse invitent les mécontentes à se désabonner. Dans les faits, il est difficile de savoir les impacts réels de cet événement sur la marque de TFPJ.

\section{Les voix de l'expérience}

La publication du billet de blogue sur les RS de TFPJ a généré des commentaires laissant entrevoir l'expérience des femmes en regard de l'alimentation des nourrissons. On remarque principalement que ce sont les femmes qui ont initié l'allaitement pour ensuite utiliser des préparations qui se sont exprimées autour de trois dimensions : 1) se reconnaître dans l'histoire de l'autre ; 2) le choix en matière du mode d'alimentation ; et 3) les sentiments associés à leur expérience.

\section{Se reconnaître dans l'histoire de l'autre}

Dans notre corpus, plusieurs femmes ont raconté leurs expériences et les émotions ressenties en regard de l'alimentation de leur nourrisson. Quelques femmes qui ont allaité avec succès ou qui ont surmonté leurs difficultés ou qui tirent leur lait témoignent de leurs expériences sous ce billet en mentionnant se reconnaître dans l'histoire de l'influenceuse.

Je me suis reconnue là-dedans!! J'allaite ma fille depuis 9 mois et demi, mais oh là là que ce n'est pas toujours facile!! À ses 6 mois j'ai acheté de la formule, je ne lui en ai jamais donné mais ça me sécurisait d'avoir l'option de lui en donner si jamais! [...] (émo : bonhomme qui sourit). (FB, 2) 
Bayard, Chantal et Laurence Charton. « Partenariat rémunéré entre une entreprise et une influenceuse sur les réseaux sociaux : entrecroisement des voix des femmes autour de l'alimentation des nourrissons. ॥ Nouvelle

Revue Synergies Canada, №15 (2022)

Je lis ça en tirant mon lait au bureau à la course et j'ai la petite larme à l'oeil... On veut le mieux pour nos petits, mais ce n'est vraiment pas facile tous les jours $\theta_{0}$. (Instagram, commentaire 3 ).

Toutefois, les femmes qui ont initié l'allaitement pour ensuite utiliser des préparations pour nourrissons sont les plus nombreuses à s'être exprimées sur les pages Facebook et Instagram de Trois fois par jour. Leurs expériences font ainsi écho à celle partagée par l'influenceuse dans son billet de blogue. Les internautes de ce sous-groupe sont d'ailleurs très nombreuses à remercier Marilou Bourdon de prendre la parole sur ce sujet " délicat », " chaud » et " controversé ». Certaines affirment se sentir " comprises » et " rassurées » par son témoignage. Pour exprimer leur gratitude, elles citent des passages du billet de blogue ou ajoutent des émoticônes.

« Troquer le jugement pour un peu plus d'amour » j'adoooore (émo: bonhomme jaune deux cœurs rouges à la place des yeux) (FB, commentaire 17)

Très beau texte. C'est tellement un choix personnel, que ça me fait de la peine de voir que des femmes qui décident de ne pas allaiter se sentent coupables. L'allaitement, ça ne regarde personne d'autre que la mère ......(FB, commentaire 15)

II est intéressant de noter que la prise de parole de l'influenceuse semble avoir encouragé d'autres femmes à témoigner de leurs expériences. La popularité de Marilou Bourdon, sa position privilégiée, semble faciliter sa prise de parole sur un sujet que les utilisatrices considèrent «tabou ". Pourquoi est-ce le cas ? Si les Québécoises amorcent l'allaitement dans une grande proportion ( $90 \%)$, elles sont moins nombreuses à atteindre le « gold standard " recommandé par les autorités de santé publique (allaitement 6 mois exclusif). Autrement dit, les préparations pour nourrissons sont utilisées par un grand nombre d'entre elles. À la lumière de ces commentaires, il subsiste donc un écart entre la pratique qui consiste à nourrir avec des préparations et la mise en visibilité de cette pratique sur les RS. Voyons pourquoi.

\section{Choisir et justifier un mode d'alimentation}

Ainsi, dans notre corpus, un très grand nombre d'utilisatrices, qu'elles soient en faveur ou non du partenariat rémunéré, s'expriment autour de la liberté de choisir des femmes en regard du mode d'alimentation de leur enfant, une question notamment abordée dans le billet de blogue de l'influenceuse : « En terminant, je veux seulement souhaiter à toutes les femmes d'être épanouies dans leur rôle de mère et de nourrir leur bébé de la manière qui leur convient, sans pression ni culpabilité. " Au même titre que cette dernière, ces utilisatrices considèrent que les femmes sont les mieux placées pour prendre une décision. Certaines d'entre elles mentionnent avoir ressenti de la pression à opter pour l'allaitement dans un contexte où ce mode d'alimentation est privilégié par les autorités de santé publique.

Tellement! On parle de libre-choix mais quand on ne choisit pas ce que la société nous impose subtilement, on se fait juger et critiquer. (FB, commentaire 64.1)

[...] Après avoir essayé l'allaitement et le tire-allaitement sans succès, j'étais complètement vidée. Autant que j'aie vécu un sentiment de culpabilité et d'échec, autant j'ai compris que l'important était de nourrir mon bébé, point barre! Merci Marilou d'avoir écrit un texte sur le sujet si controversé (émoticône cœur rouge) (FB, commentaire 4$)$

Des utilisatrices rapportent avoir l'impression de devoir expliquer leur choix à leur entourage, mais aussi à des étrangers. De nombreuses femmes détaillent ainsi sous la publication les raisons qui les ont amenés : 1) à choisir d'emblée les préparations pour nourrissons et, 2) à effectuer une transition, volontaire ou involontaire, de l'allaitement vers les préparations pour nourrissons : cancer, épuisement, problèmes de santé mentale, prendre du temps pour soi, partage de l'activité de nourrir avec le conjoint, morphologie et poids de l'enfant, manque de lait, hospitalisation de l'enfant, etc.

J'ai dû arrêter d'allaiter mon bébé après un mois, car j'ai dû commencer une chimio que l'on retardait depuis les neuf mois de maternité. Donc de sentir un jugement en donnant la bouteille à mon bébé après le premier mois a été une des étapes les plus difficiles de tout entourant mon cancer. Bref ce message me touche, car parfois ce n'est vraiment pas une question de choix, et surtout pas d'amour. (FB, 3) 
Bayard, Chantal et Laurence Charton. « Partenariat rémunéré entre une entreprise et une influenceuse sur les réseaux sociaux : entrecroisement des voix des femmes autour de l'alimentation des nourrissons. ॥ Nouvelle

Revue Synergies Canada, №15 (2022)

En réponse aux partages de leurs expériences, certaines femmes reçoivent en retour des élans d'empathie et des confidences sous la forme de mots ou d'émoticônes d'encouragement ( Courage », « Q ", « je t'admire », " je te comprends ») de la part d'autres utilisatrices qui ont vécu des expériences similaires ou qui reconnaissent l'émotion partagée dans leur témoignage. II se crée entre ces femmes un lien qui s'ancre dans l'empathie au même titre que celles qui sont plus critiques tissent des liens qui s'ancrent dans la résistance.

\section{Sentiments associés à l'expérience de nourrir avec des préparations}

Plusieurs des femmes qui ont initié l'allaitement pour ensuite utiliser des préparations ont rapporté avoir ressenti des sentiments d'échec, de deuil ou de culpabilité dans leurs commentaires. Nourrir son enfant à l'aide d'un biberon dans l'espace public semble poser particulièrement problème. Des expériences qui ne sont pas sans rappeler celles des femmes qui allaitent dans les lieux publics (Bayard, 2014 ; Boyer, 2018 ; Beach, 2017). Même si elles n'allaitent pas, ces femmes affirment être en mesure de répondre aux besoins fondamentaux de leur nourrisson, soit « nourrir » et « aimer ».

Nourrir son enfant avec amour et tendresse, répondre à un de ses besoins fondamentaux, ça ne passe pas seulement par l'allaitement. Merci pour ce texte \#teammamandamour (FB, commentaire 5)

Wow enfin!!! L'autre jour j'ai donné un biberon de mon lait et je me suis fait jugée (j'ai entendu la fille qui passait à côté dire « my god que y'en a qui ne se soucie pas de la santé de leur bébé » (c'était mon lait, mais ça, elle ne le savait pas) ... pis l'autre jour une madame qui chiale parce que j'allaite ...sérieux on s'en va où!? (FB, commentaire 32)

Par leurs commentaires, certaines d'entre elles invitent aussi les autres utilisatrices à se mettre à la place de l'autre avant d'émettre une opinion, car leurs raisons sont multiples et leurs histoires sont parfois douloureuses ( 3 gros deuils ici " (Instagram), "L'allaitement ce n'est pas toujours comme le conte de fée que nous lisons dans les livres $\theta_{-0}$ » (Instagram)). Des utilisatrices appellent à une plus grande solidarité entre les mères. D'autres encouragent les femmes à ignorer les commentaires désobligeants et les regards obliques.

\section{Les voix de l'approbation}

Enfin, près du tiers des utilisatrices sur Facebook et plus du deux tiers sur Instagram formulent un commentaire pour signifier leur approbation avec le contenu du billet de blogue à l'aide de courts énoncés jumelés à une émoticône et/ou au nom d'une personne de leur entourage (tag).

\section{Signifier leur approbation avec le contenu}

Au même titre que les femmes qui expriment leur mécontentement, un grand nombre d'utilisatrices mentionnent leur approbation avec le contenu du billet de blogue sans toutefois se positionner sur le caractère commercial du partenariat ou la gestion de la communauté par TFPJ. Ainsi, à la différence des premières, ces dernières développent généralement peu leur argumentation préférant signifier leur accord avec le contenu du billet de blogue sous la forme de courts énoncés ("Tellement vrai », " Merci », "Bien d'accord »), jumelés à une

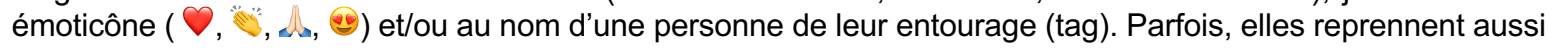
une phrase de billet de blogue.

"Troquer le jugement pour un peu plus d'amour" c'est wow! [Tag \#1] bel article à lire! [O] (FB, commentaire 79).

Le dernier paragraphe est plus que parfait il est ( il devrait ) être la raison d'être !!!! @ @mariloubiz juste wow ... continue de parler haut et fort $b$. \#onestquipourjuger $d$ (Instagram, commentaire 20)

Magnifique, j'ai des frissons » (Instagram, commentaire 7)

Contrairement aux femmes de la section précédente, ces utilisatrices ne mobilisent pas leurs expériences de femmes qui ont allaité ou qui ont utilisé des préparations pour nourrissons.

En plus des commentaires sous les publications Facebook et Instagram, les internautes ont aussi signifié leurs accords avec le contenu du billet de blogue à l'aide des fonctions disponibles pour réagir à une publication. La 
Bayard, Chantal et Laurence Charton. « Partenariat rémunéré entre une entreprise et une influenceuse sur les réseaux sociaux : entrecroisement des voix des femmes autour de l'alimentation des nourrissons. ॥ Nouvelle

Revue Synergies Canada, №15 (2022)

publication Facebook a suscité $3,2 \mathrm{~K}$ réactions, dont $92 \%$ sont positives puisque 2000 utilisatrices ont « aimé » la publication et 963 l'ont « adoré $Q$ ». Sur FB, la publication a aussi été partagée 261 fois. Toutefois, il n'est pas possible de savoir si le partage s'accompagne d'un commentaire positif ou négatif. Par ailleurs, sur Instagram, 5063 utilisatrices ont aimé la publication. II est possible de penser que certaines des réactions positives, sur Facebook ou Instagram, proviennent de fans qui « aiment » tous les contenus de la bloggeuse, indépendamment du sujet.

Enfin, en jumelant les commentaires des sections « expériences » et « approbation » aux réactions positives sur Facebook et Instagram, on constate que la majorité des personnes ont exprimé une opinion positive à l'égard du billet de blogue.

\section{Discussion}

Notre analyse des commentaires des internautes partagés sur les réseaux sociaux (Facebook, Instagram) dans le cadre du partenariat rémunéré entre Enfamil Canada et Trois fois par jour a permis de rendre visible les voix des femmes qui s'entrecroisent, s'entrechoquent, se rencontrent et se répondent autour de l'alimentation des nourrissons. Ces voix - celles du "mécontentement ou de la contestation ", de "l'expérience » ou de l' " approbation » -, qui coexistent sous une même publication, laissent entrevoir certains enjeux relatifs aux normes en matière d'alimentation des nourrissons en contexte québécois.

On remarque ainsi que les voix du « mécontentement ou de la contestation » s'appuient principalement sur des arguments autour du caractère légal du partenariat rémunéré et de la gestion transparente d'une communauté en ligne. Les femmes qui contestent ce type de partenariat s'inscrivent dans une longue tradition de militantisme qui dénonce les stratégies de marketing de l'industrie des préparations pour nourrissons depuis le boycott de Nestlé en 1977 (Nathoo et Ostry, 2009). Elles s'opposent ainsi à une industrie, dont les revenus sont estimés à 70 milliards de dollars américains (van Tulleken, 2020), qui diversifient ses pratiques de marketing en ligne pour rejoindre directement les femmes enceintes et les nouvelles mères (Abrahams, 2012; Tanrikulu et al., 2020), ce qui contrevient au Code de l'OMS. Ces utilisatrices font entendre leurs voix pour dénoncer une pratique qu'elles jugent « déloyale » et nocive ${ }^{14}$ puisqu'elles causent du tort à la santé des nourrissons et des mères. Par le fait même, ces voix participent au renforcement des normes existantes en matière d'alimentation des nourrissons en argumentant qu'il est préférable d'allaiter (6 mois exclusif) plutôt que nourrir à l'aide de préparations; et qu'il faut interdire la commercialisation et la publicité (Code) de ce type de produit. De par leurs commentaires, ces femmes manifestent leur mécontentement en se positionnant comme les gardiennes du bien-être du nourrisson. Toutefois, ces voix engagées sont notamment contestées par d'autres femmes qui affirment que le témoignage de Marilou Bourdon a favorisé leur prise de parole sur leurs expériences de nourrir à l'aide des préparations dans une société qui fait la promotion de l'allaitement, ou qui considèrent que les « contestataires » ne présentent qu'un seul côté de la médaille. Ainsi, on remarque que, comme le soulignaient Bonilla et Rosa (2015), les voix des femmes qui s'expriment dans les publications en ligne (comme hors ligne) sont multiples et révèlent des enjeux personnels et sociaux différents. Ce type de partenariat rémunéré sur les réseaux sociaux soulève par ailleurs des questions quant à l'identification et la transparence de la marque sur les réseaux sociaux, de même que sur les façons de gérer les commentaires lorsque le partenariat fait l'objet de critiques (exemple : effacement de commentaires). Enfin, pour certaines utilisatrices, il semble que, plus que la dénonciation du partenariat rémunéré entre l'influenceuse et une entreprise commerciale, ce soit le sujet des « préparations pour nourrisson " qui pose problème. On note d'ailleurs que d'autres partenariats rémunérés ont déjà été menés entre l'influenceuse et des entreprises (exemples : une entreprise de rénovation, une association qui fait la promotion des fromages du Québec, etc.) sans qu'aucune polémique n'ait lieu. Ce constat nous laisse ainsi à penser que l'alimentation des nourrissons est un sujet plus sensible pour certaines des utilisatrices de cette page.

Parallèlement, cette recherche a aussi rendu visibles les voix des femmes qui utilisent les préparations pour nourrissons (" voix de l'expérience »). Aussi, comme l'ont souligné plusieurs utilisatrices, le témoignage de l'influenceuse Marilou Bourdon de Trois fois par jour a contribué à paver la voie au partage d'expériences similaires de la part de femmes sur un sujet qu'elles qualifient de " controversé » et de « délicat ». Comme dans l'étude de Regan et Brown (2019) sur les groupes de soutien en ligne des femmes allaitantes, l'empathie, la reconnaissance mutuelle et le soutien (sous la forme de mots d'encouragements et d'émoticônes) entre des femmes qui ont vécu des expériences semblables sont aussi manifestes dans notre corpus. De plus, les jugements entre les femmes qui utilisent des modes d'alimentation différents s'observent rarement dans les échanges sous la page de Trois fois par jour. Lorsque c'est le cas, ils se manifestent plutôt indirectement par la voix de femmes qui publient des commentaires pour rappeler que l'allaitement est le " meilleur choix ». De manière générale, nous avons plutôt remarqué que les conversations qui portent sur les expériences des 
Bayard, Chantal et Laurence Charton. « Partenariat rémunéré entre une entreprise et une influenceuse sur les réseaux sociaux : entrecroisement des voix des femmes autour de l'alimentation des nourrissons. ॥ Nouvelle

Revue Synergies Canada, №15 (2022)

femmes qui utilisent des préparations pour nourrissons se déroulent dans un contexte « d'entre soi », c'est-àdire que peu de femmes qui présentent une expérience d'allaitement exclusif, par exemple, s'expriment dans ces conversations. On retrouve plutôt la parole de femmes qui ont choisi d'emblée les préparations pour nourrissons, qui ont initié l'allaitement pour ensuite effectuer une transition vers les préparations ou encore qui utilisent les deux méthodes.

De nombreuses femmes non allaitantes rapportent néanmoins percevoir ou avoir perçu le jugement des autres dans leur vie quotidienne. On observe aussi que certaines ont vécu ou vivent douloureusement la transition de l'allaitement vers les préparations pour nourrissons. Elles rapportent avoir ressenti de la pression pour allaiter, s'être senties jugées ou vivent de la culpabilité en regard de leur décision. Ces commentaires font écho à plusieurs recherches (Símonardóttir et Gíslason, 2018 ; Fallon et al., 2016) qui soulignent notamment que les femmes qui ont amorcé l'allaitement pour ensuite utiliser des préparations pour nourrissons sont plus à risque de vivre ces sentiments. Ces résultats s'observent également dans les récits des commentaires du billet de blogue de Trois fois par jour. Aussi, nous observons, au même titre que Símonardóttir et Gíslason (2018), que les femmes de notre corpus qui commentent ce billet de blogue utilisent leurs commentaires pour résister au discours hégémonique de promotion de l'allaitement plutôt que de le remettre en question. En effet, les commentaires des utilisatrices révèlent qu'elles sont nombreuses à présenter la transition vers les préparations pour nourrissons comme un " non-choix » : cancer, épuisement, problèmes de santé mentale, etc. De cette façon, elles se préservent des critiques qui les positionnent comme des femmes qui ne sont pas conscientes des bienfaits de l'allaitement ou qui priorisent leurs besoins au détriment de ceux de leurs enfants. Elles nomment également que leur préférence pour ce mode d'alimentation n'est pas synonyme de leur incapacité d'être de " bonnes mères ", c'est-à-dire des femmes qui répondent aux besoins essentiels de leur enfant (nourrir et aimer). Ces résultats font échos aux recherches ayant été réalisées ces dernières années sur la pression d'allaiter son nourrisson (Murphy, 2000 ; Lee, 2008 ; Ludlow et al., 2012). En se positionnant de cette façon, ces femmes, au même titre que celles qui contestent, contribuent à renforcer les normes en matière d'alimentation des nourrissons. Sous cette publication, très peu de voix viennent, par exemple, déconstruire le discours actuel, nuancer les faits scientifiques présentés ou les remettre en question. Ces femmes se conforment aux normes sociales ambiantes et, contrites, expliquent aux autres internautes le cas échéant pourquoi elles ne sont pas en mesure de le faire de la « bonne » façon. De plus, le témoignage d'une expérience difficile suscite de l'empathie et de la solidarité de la part d'autres internautes qui répondent par des réactions (" J'aime » ou " J'adore ») ou par des commentaires auxquels elles ajoutent des émoticônes qui les lient les unes aux autres (les plus populaires étant: $\vartheta, \because, \hat{*})$. Bien que l'influenceuse se prononce seulement à 2 reprises pour répondre aux voix du mécontentement, sa présence numérique est beaucoup plus visible sous les témoignages des femmes. L'émoticône est principalement utilisée par TFPJ dans les conversations sur ce thème (33/37). II nous apparait que ce partenariat rémunéré fait écho à la réalité vécue par plusieurs Québécoises puisqu'il met en scène une femme qui a allaité (conformément à la recommandation), a vécu des difficultés (qui ont été surmontées) et qui cherchent la meilleure préparation pour nourrir son enfant.

Néanmoins, il demeure que certaines voix semblent avoir un poids relatif plus important que d'autres. Autrement dit, ce ne sont pas toutes les voix qui sont entendues de la même manière. D'abord, certaines personnes prennent la parole plus d'une fois, intégrant un commentaire dans plusieurs conversations. Aussi, des commentaires suscitent davantage d'interactions que d'autres (ex. likes). Dans notre recherche, la consultation de tous les profils des utilisatrices qui ont commenté la publication nous a permis de constater la quasi-absence des femmes racisées ou des femmes qui s'affichent comme un formant un couple avec une partenaire de même sexe. Ces deux éléments nous font dire que ce billet de blogue cible prioritairement des femmes à l'image de l'influenceuse : blanche, classe moyenne ou privilégiée, hétérosexuelle, avec enfants.

Dans une perspective de santé publique, les RS sont vus comme des lieux potentiels pour promouvoir l'allaitement (Pérez-Escamilla, 2020 ; Marcon et al., 2018), bien qu'ils soient rarement investis par les autorités gouvernementales. II s'agit aussi de lieux privilégiés pour mieux comprendre, d'une part, les stratégies de marketing de l'industrie qui commercialisent les préparations pour nourrissons et, d'autre part, les stratégies déployées par les lactivistes pour renforcer la norme en matière d'alimentation des nourrissons (allaitement) en s'appuyant sur les recommandations des autorités de santé publique. À la lumière de nos résultats, nous partageons la conclusion de Fallon et al. (2016) qui suggèrent que les femmes qui utilisent les préparations pour nourrissons devraient faire l'objet d'une plus grande attention de la part des autorités de santé publique de façon à protéger, au même titre que les femmes qui allaitent, leur bien-être physique et émotionnel. Comme c'est le cas avec les recherches qui portent sur l'allaitement, les voix de ces femmes devraient être entendues, étudiées, et ceci est d'autant plus vrai dans un contexte de promotion de l'allaitement. 
Bayard, Chantal et Laurence Charton. « Partenariat rémunéré entre une entreprise et une influenceuse sur les réseaux sociaux : entrecroisement des voix des femmes autour de l'alimentation des nourrissons. ॥ Nouvelle

Revue Synergies Canada, №15 (2022)

\section{Notes}

${ }^{1}$ Par normalisation de l'allaitement, ces femmes entendent que : 1) le corps des femmes produit le meilleur aliment pour l'enfant; 2) les seins sont faits pour nourrir; 3) l'allaitement s'inscrit dans la continuité des processus physiologiques de la grossesse et de l'accouchement.

${ }^{2}$ L'allaitement exclusif durant les six premiers mois de la vie de l'enfant et sa poursuite jusqu'à deux ans avec l'ajout d'aliments complémentaires sont recommandés par l'Organisation mondiale de la santé (OMS) (OMS 2003; 2020), par les autorités gouvernementales canadiennes (Wall, 2001 ; PHAC, 2020) et québécoises (MSSS, 2015, 2017, 2018 ; INSPQ, 2019).

${ }^{3}$ Selon Facebook (2020), « le contenu de marque désigne tout contenu provenant d'un créateur ou d'un éditeur qui présente un partenaire commercial ou qui est influencé par un partenaire commercial, à des fins commerciales. Les créateurs comprennent les célébrités, les influenceurs ou les personnalités publiques qui publient du contenu de marque. Les éditeurs comprennent les entreprises médiatiques et entités qui publient du contenu de marque. Les partenaires commerciaux comprennent les marques, les annonceurs, les responsables marketing et les commanditaires qui commanditent du contenu de marque »: https://frca.facebook.com/business/help/213206196141623?id=491898788154026 (consulté le 31 octobre 2020).

${ }^{4}$ Depuis quarante ans, le Code et ses modifications subséquentes (IBFAN, 2019) ont pour objectif d'encadrer les pratiques de commercialisation des substituts du lait maternel dans le but de « contribuer à procurer aux nourrissons une nutrition sûre et adéquate en protégeant et encourageant l'allaitement au sein et en assurant une utilisation correcte des substituts du lait maternel » (OMS, 1981). Il s'agit d'un Code volontaire (MAQ, 2020). Au niveau international, les compagnies qui ne respectent pas le Code sont peu sanctionnées (Pérez-Escamilla, 2020). Le respect du Code est l'une des conditions pour obtenir la certification "Initiative des amis des bébés » $(I A B)$, une des principales stratégies du gouvernement québécois pour créer des milieux de soins où l'allaitement maternel est la norme (MSSS, 2015, 2017, 2018).

${ }^{5}$ La page Facebook et le compte Instagram de Trois fois par jour affichaient respectivement $479 \mathrm{~K}$ abonné.e.s et 330k abonné.e.s au moment d'écrire ces lignes (19 octobre 2020). Le billet de blogue partagé sur ces deux plateformes est intégré au site internet Trois fois par jour. Ces plateformes sont principalement suivies par des femmes âgées en moyenne entre 20 et 35 ans.

${ }^{6}$ Une entreprise québécoise spécialisée dans le domaine de l'alimentation et de l'art de la table qui partage des contenus sur diverses plateformes (site Internet, magazines en kiosque, livres, réseaux socionumériques) et vend ses produits dans deux boutiques (en ligne, pignon sur rue).

${ }^{7}$ Outre le billet de blogue, ce partenariat compte deux autres publications commanditées par Enfamil Canada qui apparaissent également sur les comptes Facebook et Instagram de TFPJ (« Burgers de saumon à l'avocat \& sauce au paprika fumé à la lime » (26 juin 2019), « Tartare de saumon au gingembre \& sésame » (27 juin 2019)).

${ }^{8}$ Marque déposée appartenant à la compagnie Mead Johnson Nutrition qui commercialise des préparations pour nourrissons sous différents formats qui sont vendus en pharmacie et dans les grandes surfaces.

9 Pour consulter le billet de blogue: https://www.troisfoisparjour.com/fr/chroniques/nourrir-son-bebe-sansculpabilite/

${ }^{10}$ Les émoticônes sont des « pictogrammes dessinés (comme les emojis : (ㅇ, , : , , etc.) ou constitués de signes issus de la banque de caractères ASCII (à lire en penchant la tête vers la gauche, pour la version « occidentale », par exemple :-), :-(, :-D, ou de face, pour la version « orientale », par exemple ${ }^{\wedge}$, O__o, ^__-^, etc.) » (Halté, 2020).

${ }^{11}$ Un hashtag est un mot ou une expression précédée d'un \#.

${ }^{12}$ Le billet de blogue a également été publié sur les RS d'Enfamil Canada, récoltant cependant peu d'attention (9 réactions sur Facebook et n'a pas été publiée sur Instagram). 
Bayard, Chantal et Laurence Charton. « Partenariat rémunéré entre une entreprise et une influenceuse sur les réseaux sociaux : entrecroisement des voix des femmes autour de l'alimentation des nourrissons. ॥ Nouvelle

Revue Synergies Canada, №15 (2022)

${ }^{13}$ Le sexe et le genre ont été attribués à partir de la photographie et/ou du prénom de la personne.

${ }^{14}$ Bien qu'observée sur le « terrain », la stratégie de marketing associant les entreprises qui vendent des préparations pour nourrissons aux influenceurs est encore peu étudiée. Nous préparons actuellement un article qui présentera de manière plus détaillée cette stratégie. Le Mouvement Allaitement Québec a produit la brochure " Protégeons l'allaitement. Les sites web et les blogues ont un rôle à jour " (2020): https://mouvementallaitement.org/environnements-favorables/code-de-commercialisation/ (consulté le 14 novembre 2020). 
Bayard, Chantal et Laurence Charton. « Alimentation des nourrissons : entrecroisement des voix des femmes sur les réseaux socionumériques. » Nouvelle Revue Synergies Canada, №15 (2022)

\section{Bibliographie}

Abrahams, S. W. (2012). Milk and social media: Online communities and the international code of marketing of breast-milk substitutes. Journal of Human Lactation, 28(3), 400-406.

Appleton, J., Laws, R., Russell, C. G., Fowler, C., Campbell, K. J. et Denney-Wilson, E. (2018). Infant formula feeding practices and the role of advice and support : An exploratory qualitative study. BMC Pediatrics, 18(12), 1-11.

Arnold, A. (2016). Voix. Dans : Juliette Rennes (Éd.), Encyclopédie critique du genre (pp. 713-722). Paris: La Découverte. https://doi.org/10.3917/dec.renne.2016.01.0713

Baillargeon, D. (2004) Un Québec en mal d'enfants. La médicalisation de la maternité, 1910-1970. Les Éditions du Remue-Ménage.

Bayard, C. (2014). "Cachez ce sein que je ne saurais voir" : Comment négocier l'allaitement dans l'espace public ?. Dans C. Bayard et C. Chouinard (Éds.) La promotion de l'allaitement maternel au Québec. Regards critiques (p. 115-135). Les Éditions du Remue-Ménage.

Beach, S. (2017). The Embodiment and Discourses of Taboo: \#brelfie. Kaleidoscope: A Graduate Journal of Qualitative Communication Research, 16(4), 43-49.

Bernard, A. (2019). Theory of the Has\#tag. Polity Press.

Bonilla, Y. et Rosa, J. (2015). \#Ferguson: Digital Protest, Hashtags Ethnography, and The Racial Politics on Social Media in the United States. American Ethnologist, 42(1), 4-17.

Boon, S. et Pentney, B. (2015). Virtual lactivism: Breastfeeding selfies and the performance of motherhood. International Journal of Communication, 9, 1759-1774.

Boyer, K. (2018). The emotional resonances of breastfeeding in public: The role of strangers in breastfeeding practice. Emotion, Space and Society, 26, 33-40.

Bridges, N., Howell, G. et Schmied, V. (2018). Exploring breastfeeding support on social media. International Breastfeeding Journal, 13(22).

Buller, R. E. (2016). Performing the breastfeeding body: Lactivism and art interventions. Studies in the Maternal, 8(2), 1-15. DOI: https://doi.org/10.16995/sim.225

Callaghan, J. E.M. et Lazard, L. (2011). 'Please don't put the whole dang thing out there!': A discursive analysis of Internet discussions around infant feeding. Psychol Health, 27(8), 938-955.

Charton, L. et de Pierrepont, C. (2018). Les forums de discussion sur les prénoms d'enfants : Entre prescriptions et normativités. Enfances Familles Générations, 31.

Facebook. (2020). À propos du contenu de marque. https://frca.facebook.com/business/help/213206196141623?id=491898788154026

Fallon, V., Komninou, S., Bennett, K. M., Halford, J. C. G. et Harrold, J. A. (2016). The emotional and practical experiences of formula-feeding mothers. Maternal \& Child Nutrition, 13(4).

Gibbs, M., Meese, J., Arnold, M., Nansen, B. et Carter, M. (2015) . \#Funeral and Instagram: Death, social media, and platform vernacular. Information Communication \& Society, 18(3), 255-268.

Giles, F. (2018). Images of women breastfeeding in public: Solitude and sociality in recent photographic portraiture. International Breastfeeding Journal, 13(52).

Grant, A. (2016). "\#discrimination” : The online response to a case of breastfeeding mother being ejected from a UK retail premises. Journal of Human Lactation, 32(1), 141-151. 
Bayard, Chantal et Laurence Charton. « Alimentation des nourrissons : entrecroisement des voix des femmes sur les réseaux socionumériques. » Nouvelle Revue Synergies Canada, №15 (2022)

Halté, P. (2020). Les émoticônes : De la signification des affects aux stratégies conversationnelles. Communiquer, 28, 19-33.

Hill Collins, P. (2016). La pensée féministe noire. Les Éditions du Remue-Ménage.

Infant Baby Food Action Network. (2019). Code international de commercialisation des substituts du lait maternel. Résolutions subséquentes de l'Assemblée mondiale de la santé. https://www.ibfan.org/. Consulté le 8 novembre 2020.

Institut national de santé publique. (2019). Allaitement. Https://mobile.Inspq.Qc.Ca/informationperinatale/fiches/allaitement. Consulté le 8 novembre 2020.

Jarty, J. et Fournier, T. (2020) 'Healthy children, healthy Nations'. Discipliner les corps reproducteurs pour la santé de qui ?. Enfances Familles Générations, 33.

Kukla, R. (2006). Ethics and ideology in breastfeeding advocacy campaigns. Hypatia, 21(1), 157-180.

Leaver, T., Highfield, T. et Abidin, C. (2020). Instagram. Polity Press.

Lee, E. J. (2008). Living with risk in the age of 'intensive motherhood': Maternal identity and infant feeding. Health, Risk \& Society, 10(5), 467-477.

Locatelli, E. (2017). Images of breastfeeding on Instagram: Self-representation, publicness, and privacy management. Social Media + Society, 3(2).

Ludlow, V., Newhook, L. A., Newhook, J. T., Bonia, K., Goodridge, J. M. et Twells, L. (2012). How formula feeding mothers balance risks and define themselves as 'good mothers'. Health, Risk \& Society, 1(14), 291-306.

Marcon, A. R., Bieber, M. et Azad, M. B. (2018). Protecting, promoting, and supporting breastfeeding on Instagram. Maternal \& Child Nutrition, 15(1).

Mecinska, L. (2018). Embodied online activism: Breastfeeding activism (lactivism) on Facebook. Networking Knowledge: Journal of the MeCCSA Postgraduate Network, 11(2), 21-38.

Ministère de la Santé et des Services Sociaux. (2015). Programme national de santé publique 2015-2025, Gouvernement du Québec.

Ministère de la Santé et des Services Sociaux. (2017). Code international de commercialisation des substituts du lait maternel. https://www.msss.gouv.qc.ca/professionnels/perinatalite/amis-des-bebes/codeinternational-de-commercialisation-des-substituts-du-lait-maternel/ Consulté le 30 octobre 2020.

Ministère de la Santé et des Services Sociaux. (2018). Politique de périnatalité 2008-2018 », Gouvernement du Québec.

Morrissey, M. E. et Kimball, K. Y. (2017). \#SpoiledMilk: Blacktavists, visibility, and the exploitation of the black breast. Women's Studies in Communication, 40(1), 48-66.

Moukarzel, S., Rehm, M. et Daly, A. J. (2020). Breastfeeding promotion on Twitter: A social network and content analysis approach. Maternal \& Child Nutrition, 16(4).

Mouvement Allaitement Québec. (2020) Protégeons l'allaitement. Les sites web et les blogues ont un rôle à jour. https://mouvementallaitement.org/environnements-favorables/code-de-commercialisation/. Consulté le 8 novembre 2020.

Murphy, E. (2000). Risk, responsibility, and rhetoric in infant feeding. Journal of Contemporary Ethnography, 29(3), 291-325.

Nathoo, T. et Ostry, A. (2009). The one best way? Breastfeeding history, politics, and policy in Canada. Wilfrid 
Bayard, Chantal et Laurence Charton. « Alimentation des nourrissons : entrecroisement des voix des femmes sur les réseaux socionumériques. » Nouvelle Revue Synergies Canada, №15 (2022)

Laurier University Press.

Niela-Vilén, H., Axelin, A., Melender, H-L. et Salantera, S. (2015). Aiming to be a breastfeeding mother in a neonatal intensive care unit and at home: A thematic analysis of peer-support group discussion in social media. Maternal \& Child Nutrition, 11, 712-726.

Orchard, L. et Nicholls, W. (2020). A systematic review exploring the role of social media in breastfeeding decision making, promotion, and support. Preprint. DOI:10.31234/osf.io/bxrf7

Organisation mondiale de la santé. (1981). Code international de commercialisation des substituts du lait maternel https://www.msss.gouv.qc.ca/professionnels/perinatalite/amis-des-bebes/code-internationalde-commercialisation-des-substituts-du-lait-maternel/. Consulté le 14 novembre 2020.

Organisation mondiale de la santé. (2003). Stratégie mondiale pour l'alimentation du nourrisson et du jeune enfant. Genève : OMS.

Organisation mondiale de la santé. (2020). Allaitement. https://www.who.int/topics/breastfeeding/fr/. Consulté le 25 février 2020.

Pérez-Escamilla, R. (2020). Breastfeeding in the $21^{\text {st }}$ century: How we can make it work. Social Science \& Medicine, 244.

Public Health Agency of Canada. (2020). 10 Great Reasons to Breastfeed your Baby. https://www.canada.ca/en/public-health/services/health-promotion/childhood-adolescence/stageschildhood/infancy-birth-two-years/breastfeeding-infant-nutrition/10-great-reasons-breastfeed-yourbaby.html. Consulté le 14 novembre 2020.

Rabatel, A. (2012). Les relations locuteur/énonciateur au prisme de la notion de voix. Arts et savoirs, 2.

Regan, S. et Brown, A. (2019). Experiences of online breastfeeding support: Support and reassurance versus judgement and misinformation. Maternal \& Child Nutrition, 15(4). DOI: https://doi.org/10.1111/mcn.12874

Rippey, P. L. F. (2021). L'allaitement et la recherche du bonheur : comment l'alimentation du nourrisson est passé d'un acte mondain à un acte moral. Dans L. Charton et C. Bayard (éds.) Des imaginaires aux réalités conjugales familiales. Perspectives interdisciplinaires et internationales. Les Presses de I'Université du Québec.

Símonardóttir, S. et Gíslason, I. V. (2018). When breast is not best: Opposing dominant discourses on breastfeeding. The Sociological Review, 66(3), 665-681.

Skelton, K. R., Evans, R., LaChenaye, J., Amsbary, J., Wingate, M. et Talbott, L. (2018). Exploring social media group use among breastfeeding mothers: Qualitative analysis. JMIR Pediatr Parent, 5(1-2). DOI : https://doi.org/10.2196/11344

Tanrikulu, H., Neri, D., Robertson, A. et Mialon, M. (2020). Corporate political activity of the baby food industry: The example of Nestlé in the United States of America. International Breastfeeding Journal, 15(22).

Vallières, A. (2021). « C'était pas mal plus difficile dans ma tête ». L'idéalisation de l'allaitement maternel et les influences sur le vécu des mères primipares. Dans L. Charton et C. Bayard (éds.) Des imaginaires aux réalités conjugales familiales. Perspectives interdisciplinaires et internationales. Les Presses de I'Université du Québec.

Van Tulleken, C., Wright C., Brown A, Mc Coy D. et A. Costello. (2020). Marketing of breastmilk substitutes during the COVID-19 pandemic. The Lancet, 396(10259), p. e58. https://www.sciencedirect.com/science/article/pii/S014067362032119X Consulté le 14 novembre 2020.

Wall, G. (2001). Moral constructions of motherhood in breastfeeding discourse. Gender \& Society, 15(4), 592610. 
Bayard, Chantal et Laurence Charton. «Alimentation des nourrissons : entrecroisement des voix des femmes sur les réseaux socionumériques. » Nouvelle Revue Synergies Canada, №15 (2022)

Willig, C. (2014). Discourses and Discourse Analysis. Dans U. Flick (dir.), The SAGE Handbook of Qualitative Data Analysis (p. 341-353). SAGE Publications. 